\title{
From spears to speech
}

\section{Could throwing spears have laid the foundations for language acquisition?}

\section{A Brief History of the Mind: From Apes to Intellect and Beyond \\ by William $\mathrm{H}$. Calvin \\ Oxford University Press: 2003. \$26}

\section{Robin Dunbar}

After a century of neglect, the mind has suddenly become an issue of evolutionary interest once again. Partly thanks to evolutionary psychology (by which I don't mean the pastiche that the media has largely been responsible for creating), the differences between human and non-human minds and their origins have begun to spawn a number of books. Notwithstanding the enthusiasm in the 1970s and 1980s for the similarities between humans and our primate cousins, both in popular culture and among academics, the fact is that humans are very different from even our ape sister species. William Calvin's latest book looks at how different we really are.

The essence of Calvin's argument is that the difference between humans and other animals comes down to what he calls "structured stuff" (that is, coordinated, structured task processing). One of the most obvious examples is the way we deconstruct sentences to expose their meaning. We can do this, he argues, because we evolved the capacity to coordinate fine-tuned movements in the context of throwing. The great revolution in human evolutionary history stems from the shift from the older forms of heavy-duty hunting, mostly by dint of thrusting spears, to projectile hunting (throwing spears or using bows), which required careful aiming and much finer coordination.

Practice at these activities fine-tuned the neural machinery that allowed the delicate motor control required for speech and language. Much is made, in this respect, of the growing evidence for the brain's ability to coopt neural circuits. For example, the neural substrates for reading have different location in the brain in different individuals, as one might expect of a skill that does not have a long evolutionary history. This 'softwiring', as Calvin calls it, is clearly of major importance in human cognition.

I found the themes of the book, broadly speaking, congenial, and the account well informed and authoritative, as one might expect from a neuroscientist and science popularist of Calvin's stature. However, there are aspects of this particular book that I found less satisfying. Calvin's insistence on the importance of a gesturally based phase to language evolution does not, I think, make sense. Language is a parsing skill, and, even though parsing is a hierarchical process, it seems to me to be a very different kind of skill from that used in coordinated throwing. Manipulating concepts is not the same kind of activity as manipulating muscle masses. Nor does the timing really work. The evidence, as Calvin himself notes, points to a period about 500,000 years ago as the likely timing for the origin of speech, if not full-blown language. But the archaeological record is very clear that real projectilebased hunting did not become widespread until the Upper Palaeolithic revolution, which kicked in around 50,000 years ago (perhaps a little earlier in Africa). The evolution of speech, then, pre-dates the fine muscle control of aimed throwing by a very wide margin.

I also found the writing style somewhat irksome. Of course, this is a book intended for a popular audience, and perhaps a certain kind of popular audience appreciates this kind of erratic, scattergun style of writing. I just find it irritating. The last chapter is an attempt to look into the future, our future. I found it largely unconvincing. There are substantive issues about the extent to which the natural limits to human cognitive flexibility might constrain our ability to cope with the stresses and strains of the modern urban jungle, or with the opportunities offered by the information highway. These issues are of genuine interest and real significance. But I saw none of them discussed here.

Robin Dunbar is at the School of Biological Sciences, Biosciences Building, Crown Street, University of Liverpool, Liverpool L69 7BZ, UK.

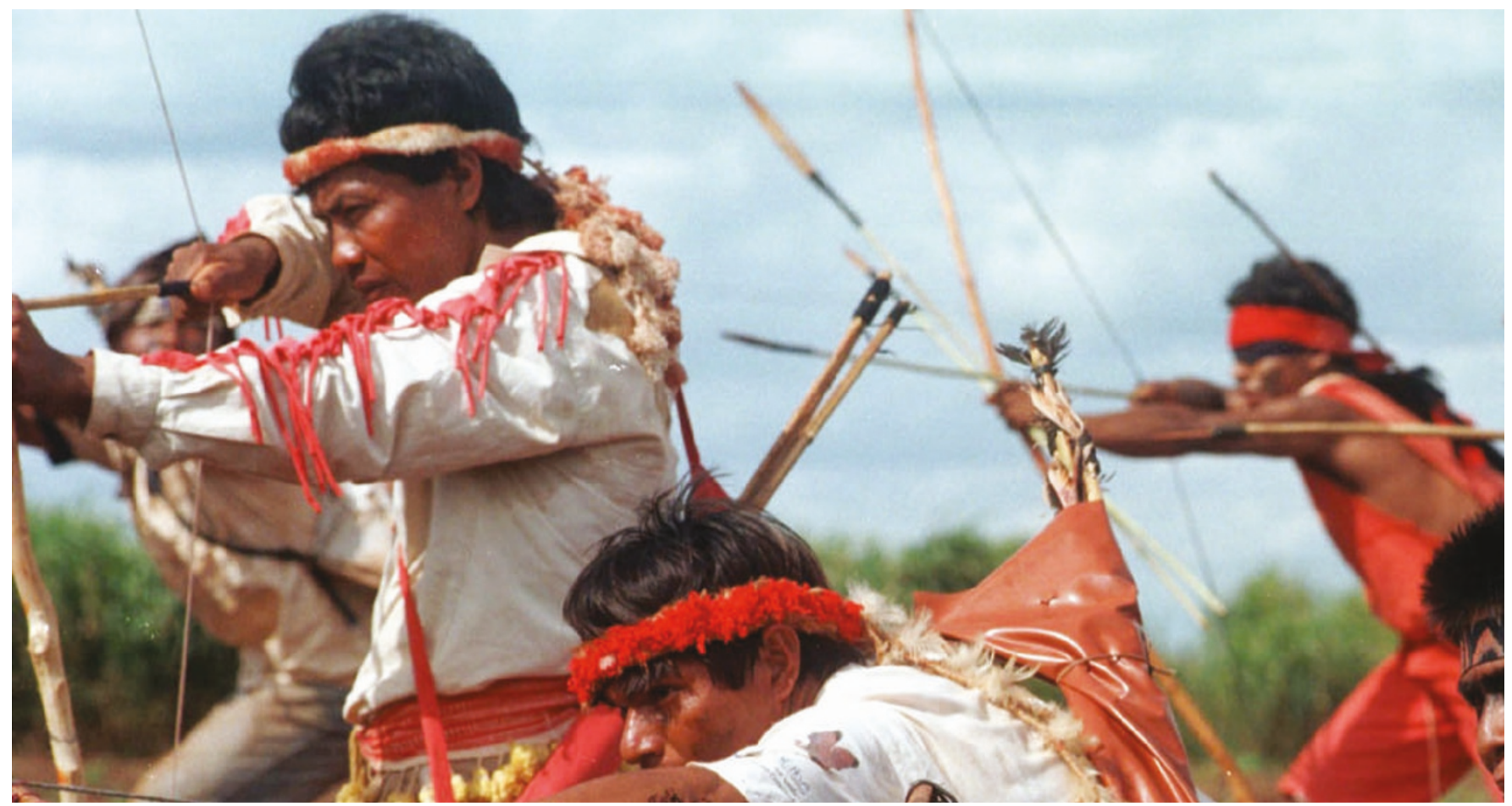

That's fighting talk: the neural control of the muscles required for firing arrows is similar to that needed to control speech. 